\title{
ถ⿻上丨์ \\ INTERNACIONAL \\ DEESESON \\ EDIÇĀO 2017 \\ Órtese e Elementos Finitos: Um Estudo Para o Desenvolvimento de Novos Designs Através de Manufatura Aditiva
}

\author{
Mariana Ribeiro Volpini Lana; \\ Anderson Horta; \\ Marco Borges; \\ Paulo Reis;
}

resumo:

O padrão de caminhada em indivíduos que apresentam disfunções musculares fomenta a demanda por dispositivos auxiliares de locomoção. Estes artefatos de assistência ao movimento variam de acordo com sua forma, tamanho e características funcionais, obedecendo às aplicações clínicas desejadas. Dentre as órteses de membro inferior, o Tutor Curto visa melhorar a capacidade do andar em indivíduos com limitações neuromusculares diversas, oferecendo níveis variados de suporte durante a marcha. $\mathrm{O}$ objetivo deste estudo é explorar a possibilidade de utilização de novos designs em Manufatura Aditiva para reproduzir a forma e as características funcionais de um Tutor Curto de forma eficaz. Para tanto, este trabalho apresenta um estudo sobre a atuação das forças mecânicas através da análise de Elementos Finitos em um tutor curto. Será apresentado o estudo de distribuição estresse decorrente do desenvolvimento da marcha, bem como o deslocamento do dispositivo ortopédico no decorrer da caminhada do paciente.

palavras-chave: manufatura aditiva, novos designs órteses, elementos finitos. 


\section{Introdução}

De acordo com o relatório do Instituto Brasileiro de Geografia e Estatística (IBGE) em 2014, aproximadamente 8 milhões de indivíduos no Brasil apresentam alguma deficiência relacionada a limitações motoras nos membros inferiores (IBGE, 2014).Considerando este cenário, houve expressivo aumento na utilização de órteses para tratar disfunções neuromotoras com intuito de gerar alinhamento biomecânico e consequente prevenção de encurtamentos musculares, deformidades ósseas e melhora da funcionalidade da marcha de pacientes (DEBERG ET AL, 2014; KELLY ET AL 2007).

Utilizado para o tratamento das disfunções motoras relacionadas aos membros inferiores, o tutor curto é um dispositivo terapêutico auxiliar na reabilitação que tem por objetivo limitar o movimento de flexão plantar do tornozelo, mantendo a articulação tibiotársica em postura rígida a $0^{\circ}$ de dorso flexão como demonstrado na Fig. 1 (Faustini, et al 2008; Milusheva, et al 2007).

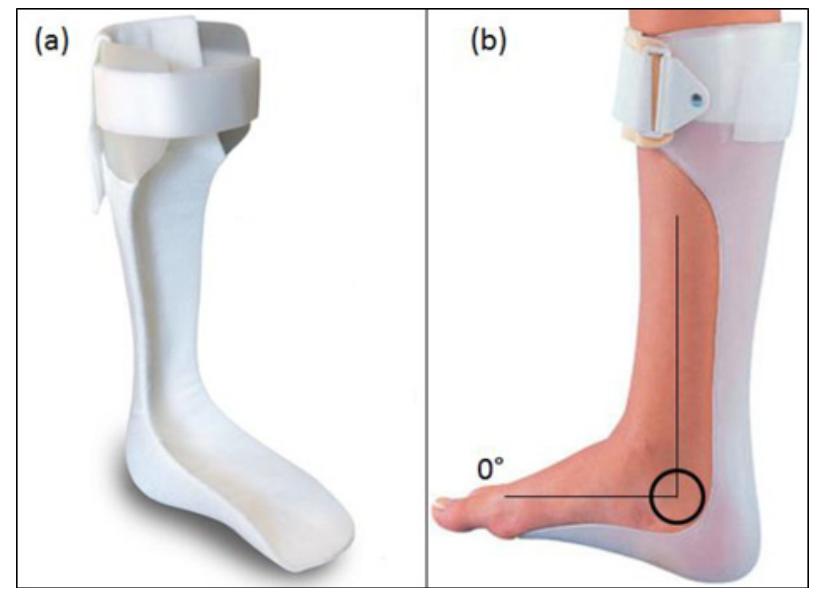

Fonte: Healt (2017); Direct (2017)

Figura 1: (a) Tutor Curto Rígido (b) Utilização do Tutor Curto

Todavia, o processo atual de fabricação dos dispositivos ortopédicos, em especial dos tutores curtos, é extremamente artesanal, demandando intensivo esforço operacional, além de apresentar limitações na flexibilização do modelo produzido, ou seja, de designs que poderiam ser oferecidos aos usuários (Pallari et al, 2010; Salles e Gyi, 2012; Salles e Gyi, 2013). Diante do exposto, faz-se necessária a procura pela utilização de novas tecnologias de fabricação de órteses capazes de gerar rapidez em etapas do processo de confecção, mantendo a qualidade do produto e o potencial em trazer benefícios para os pacientes no que diz respeito ao conforto, a praticidade de entrega do produto e à possibilidades de diversificação do design associado a uma estética contemporânea, bem como um resultado funcional e aprazível (JIN ET al, 2015).

Como possível solução para as demandas de manufatura de objetos complexos, a Manufatura Aditiva (MA), apresenta soluções factíveis para o desenvolvimento de dispositivos ortopédicos de geometria não convencional através da utilização de modelos computacionais e fabricação por deposição de camadas (FAUSTINI ET AL, 2008, SCHRANK E STANHOPE, 2011).

Contudo, para que a funcionalidade dos tutores curtos seja, de fato, correspondente com a necessidade requerida, testes preliminares devem ser realizados para aceitação das órteses de acordo com as especificações de resistência mecânica postuladas. Tais testes podem ser realizados por intermédio de ensaios computacionais baseadas em Elementos Finitos (EF) (PAWALE et al, 2012).

O presente estudo tem por objetivo abordar a aplicação de ferramentas computacionais CAD (Computer Aided Design) para auxiliar no desenvolvimento de dispositivos ortopédicos através de manufatura aditiva. O enfoque principal é a aplicação de ensaios de EF associados aos desenhos computacionais com o objetivo de elucidar e avaliar as ações resultantes de forças mecânicas sobre o tutor curto em seu modelo virtual. Os resultados dessa análise indicam as áreas de maior esforço mecânico empregadas em seu uso, o que demarca, por consequência, as regiões passíveis de retirada de material para as propostas de design, respeitando os limites devidos. 


\section{Revisão Bibliográfica}

\subsection{Manufatura Aditiva em Auxílio á Bioengenharia}

A demanda pela confecção avançada de protótipos funcionais e estruturais, promovida pela necessidade no desenvolvimento de pesquisas em manufatura e pela crescente carência dos setores industriais para oferecer uma resposta rápida às solicitações de mercado, consolidou 0 desenvolvimento do conceito de Manufatura Aditiva (RAJA E FERNANDES, 2008; VOLPATO et al., 2007).

Para Raja e Fernandes(2008) a MA se traduz na classe de tecnologias de manufaturara utilizadas na fabricação de objetos físicos a partir de deposição de material, camada por camada, tomando como referência a representação virtual do objeto por sistemas 3D-CAD. Segundo os autores, as técnicas de MA são o melhor processo de manufatura existente para o fabrico de pequenos componentes e peças complexas.

As técnicas de MA foram inicialmente empregadas na medicina no início do século XXI, com o desenvolvimento de implante dentários e próteses personalizadas (GROSS, 2014; CUI, 2012). A partir de então o desenvolvimento e utilização da impressão 3D na medicina tem se tornado uma prática constante, e tem demonstrado evolução constante. As utilizações médicas da MA podem ser organizadas em diversas categorias, como a fabricação de tecidos e órgãos; Criando próteses, implantes e modelos anatômicos; E pesquisa farmacêutica (BANKS, 2013). Atualmente, as principais aplicações observadas para os modelos e biomodelos gerados através de impressão 3D são o treinamento cirúrgico, a pesquisa e ensino na medicina, interação paciente-médico, planejamento de cirurgias, engenharia de tecidos e reabilitação ortopédica (DEBERG et al, 2014; GIBSON E CHEUNG, 2006).

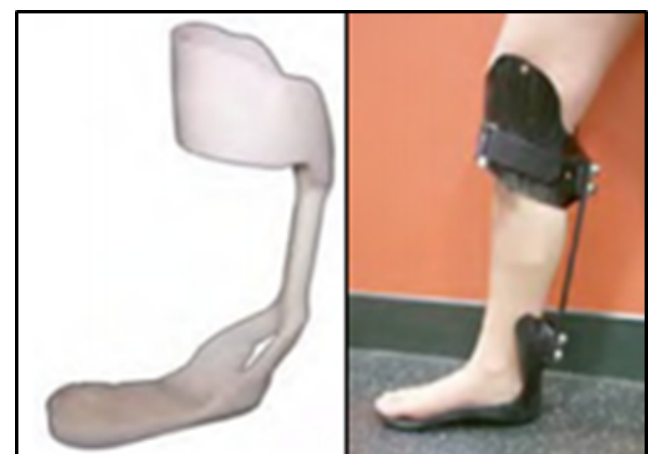

Fig. 2: Dispositivos ortopédicos fabricados por MA. (HARPER, 2014; SCHRANK, 2013)

As técnicas de MA foram inicialmente empregadas na medicina no início do século XXI, com o desenvolvimento de implante dentários e próteses personalizadas (GROSS, 2014; CUI, 2012). A partir de então o desenvolvimento e utilização da impressão 3D na medicina tem se tornado uma prática constante, e tem demonstrado evolução constante. As utilizações médicas da MA podem ser organizadas em diversas categorias, como a fabricação de tecidos e órgãos; Criando próteses, implantes e modelos anatômicos; E pesquisa farmacêutica (BANKS, 2013). Atualmente, as principais aplicações observadas para os modelos e biomodelos gerados através de impressão 3D são o treinamento cirúrgico, a pesquisa e ensino na medicina, interação paciente-médico, planejamento de cirurgias, engenharia de tecidos e reabilitação ortopédica (DEBERG et al, 2014; GIBSON E CHEUNG, 2006).

\subsection{Elementos Finitos}

A Mecânica dos Meios Contínuos, principalmente a Teoria da Elasticidade, tem como preceito básico o desenvolvimento de modelos matemáticos que possam representar adequadamente a situação física real de componentes sujeitos a esforços mecânicos. Em análise estrutural, o objetivo pode ser a determinação do campo de deslocamentos, as deformações internas ou as tensões atuantes no sistema 
devido à aplicação de cargas (AGARWAL E BROUTMAN, 1974; SERGELIND, 2013; CUI et al, 2012).

Considerando as ferramentas e métodos de resolução de equações com grande número de variáveis, a análise de Elementos Finitos (EF) se apresenta como um procedimento numérico que se objetiva em resolver equações diferenciais parciais que dificilmente podem ser solucionadas de maneira analítica (SERGELIND, 2013). O método consiste em determinar a geometria fracionada de uma superfície (Fig. 3), e, através de um sistema de equações algébricas determinar os relacionamentos entre os pontos fracionados. Em função desta subdivisão geométrica é possível testar a atuação de equações diferenciais representativas de ensaios mecânicos em superfícies com parâmetros pré-determinados, possibilitando assim estimar as propriedades mecânicas desta superfície (AGARWAL E BROUTMAN, 1974; JAKUBINEK, 2010).

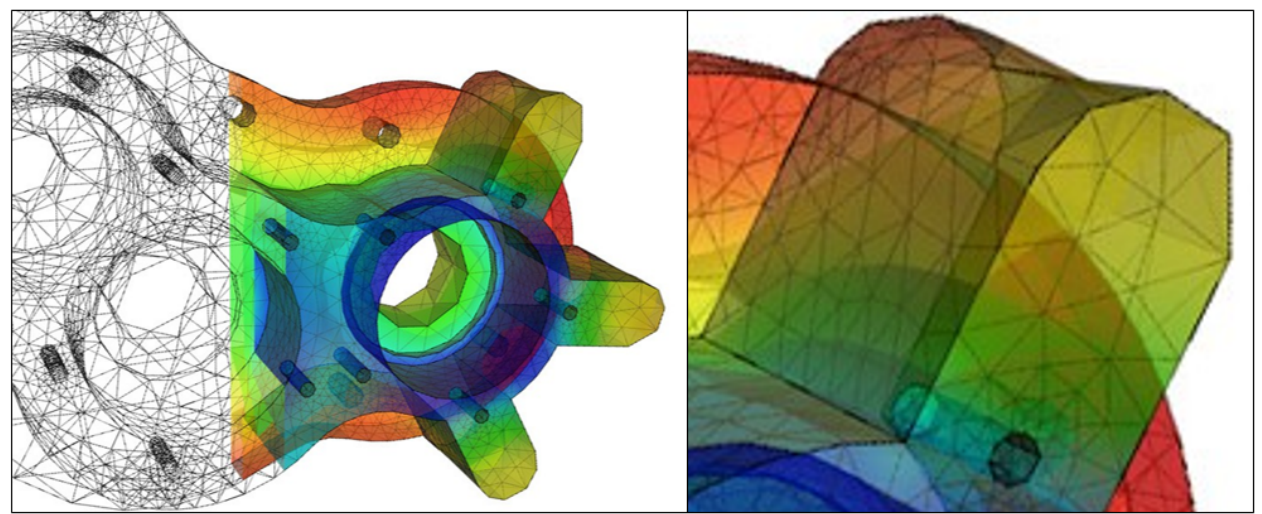

Fig. 3: Representação do fracionamento de superfície virtual em um estudo de EF. (CHEN et al., 2001)

Os sistemas de Elementos Finitos permitem analisar e testar superfícies em ambiente computacional, sem a necessidade de realização de testes destrutivos, motivo pelo qual a utilização desta ferramenta tem sido amplamente viável em ensaios necessários para simulação de forças em um dado material (CHEN et al, 2001). É importante observar que o método de EF é uma estimativa do comportamento mecânico de um determinado objeto sujeito à atuação de forças, portanto, não substitui testes mecânicos que tem por objetivo qualificar as propriedades mecânicas de um material em estudo.

A maior vantagem do teste de elementos finitos é a capacidade de representar os detalhes geométricos, materiais da estrutura, assim como a aplicação de múltiplos conceitos de projetos. A maior desvantagem deste método é o custo/ tempo, visto que desenvolver um modelo de estruturas complexas pode demandar muito tempo devido às divisões geométricas dos elementos e execução da malha computacional (GANDI, 2002).

\section{Métodos}

\subsection{Geração do Modelo Elementos Finitos}

Considerando o objetivo central deste trabalho, as ferramentas utilizadas em nível virtual se objetivaram no suprimento de dados e coordenadas espaciais para a execução do estudo de EF. Para que o objetivo fosse atingido, uma órtese foi desenvolvida através de ferramentas computacionais de modelagem de sólidos virtuais, conforme Fig. 4. Como pode ser visualizado na Fig. 4, a órtese desenvolvida possui referencias dimensionais compatíveis com a morfologia de um adulto de estatura média, com dimensões de $182.69 \mathrm{~mm}$ em Z, $99.05 \mathrm{~mm}$ em Y e $55.69 \mathrm{~mm}$ em X. A atuação das forças de simulação aplicadas foram estimadas considerando a compressão do pé na órtese e a atuação das forças exercidas no tendão de Aquiles e nos tendões dos músculos flexor e extensor durante o movimento do pé, como flexão dorsal, rotação do pé e forças de compressão do triceps sural na região de contato com a órtese. A atuação das forças de contato com o solo foi simulada por forças nodais equivalentes às forças de reação. 


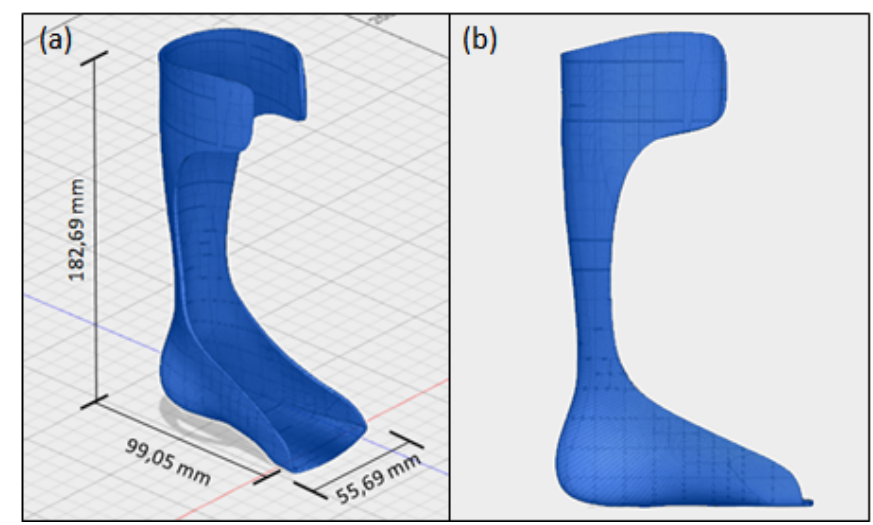

Fig. 4: (a) dimensões da órtese virtual. (b) perspectiva lateral da órtese virtual.

A partir da órtese virtual apresentada, foi desenvolvida a segmentação da superfície do modelo para a realização dos testes de EF. Os testes efetuados buscaram simular a condição de marcha do paciente usuário de tutor curto, com as forças de correção atuantes nas regiões observadas por Manee, $2011 \mathrm{em}$ seus estudos sobre a atuação corretiva da órtese suropodálica.

Os resultados obtidos para o método de Elementos Finitos neste estudo estão condicionados com design da órtese desenvolvida, com os fatores de resultantes de forças provenientes da marcha e das características físicas representadas para este trabalho. É importante ressaltar que a resistência mecânica e distribuição de stress resultantes se diferenciar dos resultados deste estudo conforme a variação dos parâmetros utilizados.

\subsection{Propriedade do Material}

O material utilizado como parâmetro para a realização dos testes de EF foi o termoplástico à base de nylon DuraForm ProX PA, desenvolvido pela fabricante 3D Systems. O material em questão apresenta como característica alta resistência e durabilidade, sendo uma base de produção funcional para peças produzidas pro MA nas industrias automotiva, aeroespacial e de bens de consumo. As especificações técnicas do material são demonstradas na Tabela 1.

TABELA I

PROPRIEDADES MECÂNICAS DO DURAFORM PROX PA (3D SYSTEM, 2017)

\begin{tabular}{|c|c|}
\hline Material Property & Value \\
\hline Density (sintered part) & $0.95 \mathrm{~g} / \mathrm{cm}^{3}$ \\
\hline $\begin{array}{l}\text { Flexural Modulus } \\
\qquad(\mathrm{MPa})\end{array}$ & 1650 \\
\hline $\begin{array}{c}\text { Flexural Strength, } \\
\text { ultimate }(\mathrm{MPa})\end{array}$ & 63 \\
\hline $\begin{array}{l}\text { Tensile Modulus } \\
\qquad(\mathrm{MPa})\end{array}$ & 1770 \\
\hline $\begin{array}{l}\text { Tensile strength, } \\
\text { Ultimate (MPa) }\end{array}$ & 47 \\
\hline Elongation at break & $22 \%$ \\
\hline Impact strength $(\mathrm{J} / \mathrm{m})$ & \\
\hline Notched Izod, $23{ }^{\circ} \mathrm{C}$ & 45 \\
\hline $\begin{array}{l}\text { Unnotched Izod, } 23 \\
{ }^{\circ} \mathrm{C}\end{array}$ & 644 \\
\hline $\begin{array}{l}\text { Heat deflection } \\
\text { temperature }\end{array}$ & $\begin{array}{l}182^{\circ} \mathrm{C} \\
97^{\circ} \mathrm{C}\end{array}$ \\
\hline Flammability & HB \\
\hline Hardness & $73 \mathrm{D}$ \\
\hline
\end{tabular}




\section{Resultados}

Como resultado experimental, a análise do método de Elementos Finitos fornece a quantificação da distribuição global de estresse e a deformação da estrutura analisa a partir da atuação da resultante de forças. Para o estudo em questão, o resultado da simulação da marcha por EF foi apresentada em duas variações observadas na realização da simulação, a saber: o deslocamento percentual da órtese na marcha e o os testes de estresse, que demonstraram as regiões de maior atuação das forças mecânicas e consequente maior desgaste da órtese. A apresentação destas variações de resultados se fazem importantes por influenciarem diretamente na atuação corretiva do dispositivo ortopédico, visto que as ações clínicas corretivas são decorrentes da rigidez da órtese. A Fig. 5 apresenta a primeira variante, deslocamento da órtese na marcha.

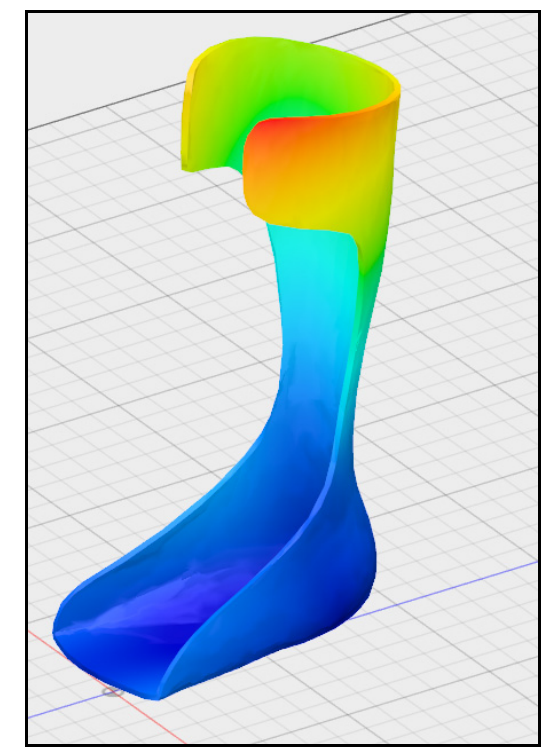

Fig. 5: deslocamento da órtese na marcha.

O maior módulo de deformação em função do deslocamento do modelo virtual da órtese é observado na região superior esquerda do dispositivo, se estendendo, com menor proporção, ao longo da haste. A maior intensidade de deslocamento foi na presilha que mantem o dispositivo fixo no membro inferior do usuário da órtese, atingindo uma deformação máxima de $65.51 \mathrm{~mm}$. $\mathrm{O}$ fato observado pode ser explicado considerando a relação da disposição estrutural da órtese no ponto citado versus a força exercida pelo membro do paciente nas presilhas de fixação. A segunda variante de resultado foi a simulação de estresse do material estudado, que pode ser observado na Fig. 6.

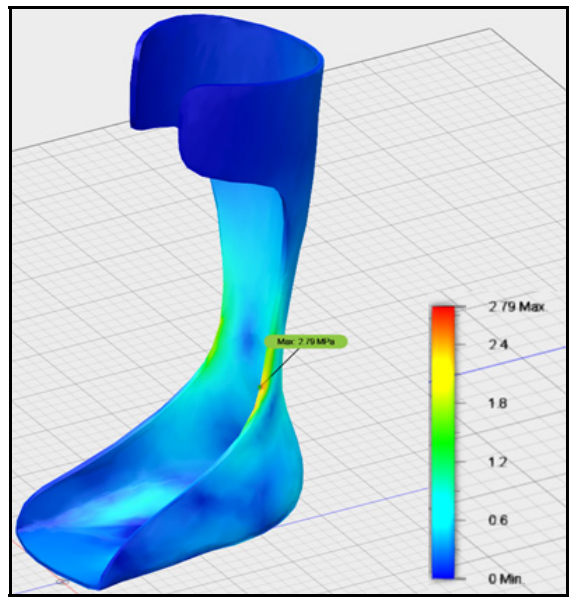

Fig. 6: Distribuição de estresse no órtese. 
O maior desgaste no modelo de estudo foi observado na região inferior da haste da órtese. Por ser uma região que demanda maior rigidez devido ás finalidades de correção do dispositivo a flexão neste ponto tem uma tolerância relativamente baixa se comparado à outras regiões da órtese. A Fig. 7 demonstra a região observada de maior estresse.

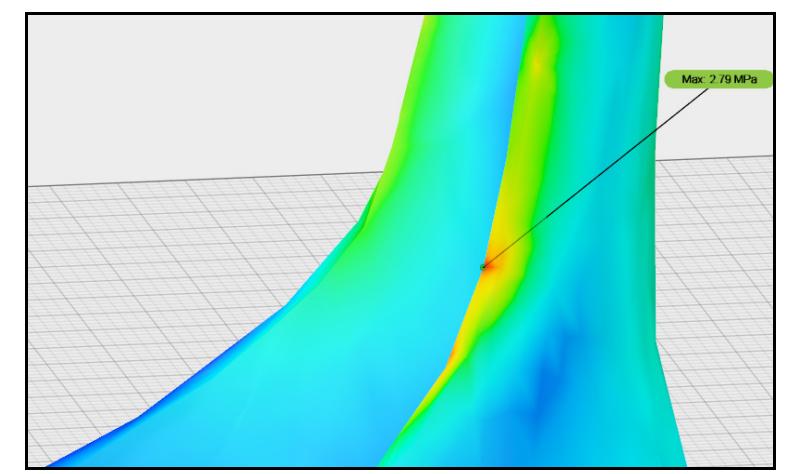

Fig. 7: Região observada de maior atuação de estresse

A região de estresse de maior atuação da resultante de forças apresentou módulo de 2,79 Mpa, e é a região mais propensa ao desgaste e consequente fratura da superfície do material.

Considerando as forças provenientes do pé atuantes durante a marcha na região plantar da órtese, a resistência ao desgaste, bem como a resistência as deformações não demonstraram valores significativos atuantes. Esse fato pode ser explicado pela maior deposição de material na região plantar da órtese no modelo analisado, o que promove propriedades mecânicas de maior importância nesta região.

.Para fins de análise comparativa o Gráfico 1 apresenta o deslocamento global relativo da órtese fabricada com o material de análise deste estudo, DuraForm ProX PA, através de MA em comparação com a órtese fabricada através pelo método tradicional a partir de polipropileno de alta densidade. Os valores observados para esta comparação são provenientes de simulações computacionais fundamentadas nos mesmo parâmetros de força e estrutura.

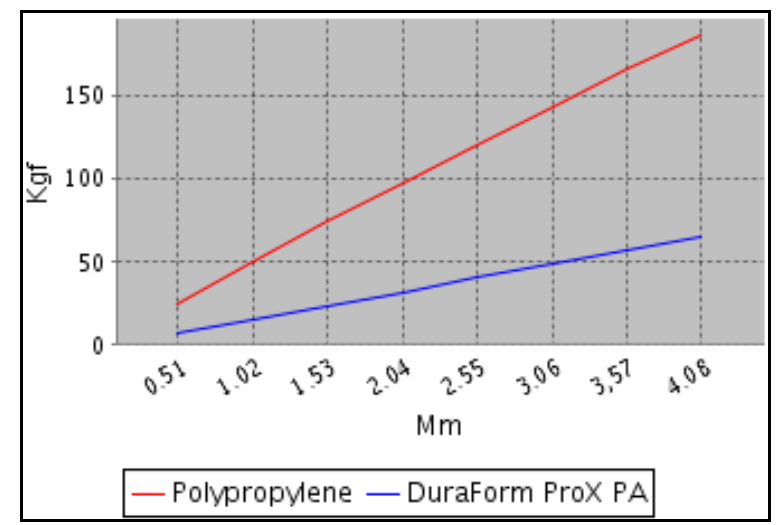

Gráfico. 1: Deslocamento global da órtese em função da resultante de forças.

Conforme observado, a órtese fabricada por MA apresentou menor deslocamento global se comparado com a órtese fabricado em polipropileno, demonstrando maior rigidez estrutural, favorecendo assim a atuação das forças corretivas necessárias para a atuação clínica do dispositivo ortopédico.

\section{Considerações Finais}

Este trabalho teve como objetivo dar suporte à atividade projetual de novos designs para Manufatura Aditiva (MA) de dispositivos ortopédicos para membros inferiores. A simulação de EF realizada apresentou relativo deslocamento e stress em determinadas regiões da órtese, todavia, as deformações e forças mecânicas observadas pela análise de EF não inviabilizam a fabricação do tutor pela técnica 
de impressão tridimensional e parâmetro de material utilizado para a realização deste estudo, pelo fato de não interferir na fixação do dispositivo no membro e não afetar a rigidez requerida para a imobilização das regiões de interesse. É importante observar que conforme o decorrer da utilização da órtese haverá maior desgaste de material na região ressaltada pela simulação de stress, região onde localizam-se os metatarsos no dispositivo, sugere-se então, maior deposição de material na região em destaque, para que a durabilidade do ciclo de utilização da órtese se prolongue.

Considerando as regiões da órtese que sofrem menor stress pelas forças aplicadas, tanto em marcha quanto em repouso, demonstradas no presente estudo, é possível visualizar e delimitar áreas onde as interferências por retirada de material não interfeririam de maneira negativa na resistência do aparelho ortopédico.

Os recursos de retirada de material para projetos de novos designs para as órteses são importantes na configuração visual e relações de conforto e praticidade de uso. Isso porque, ao contrário da adição de material poderia dificultar seu uso, principalmente considerando calçados e roupas utilizadas pelo paciente.

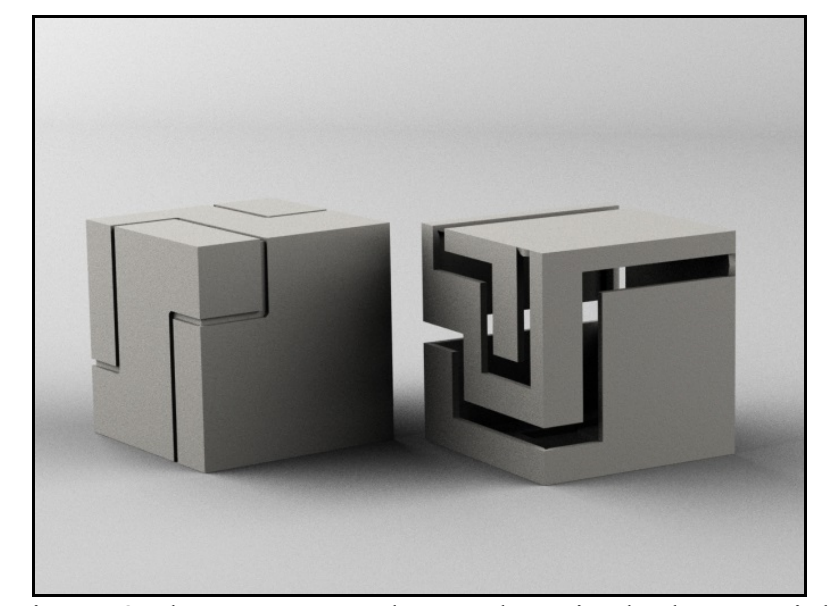

Figura 8: demonstração de uso de retirada de material

Como demonstrado na Figura 5, o uso de frisos e detalhes em baixo-relevo e perfurações de formatos específicos na superfície são parte dos citados recursos de retirada de material que permitem maior gama de alternativas para os projetos de design nas propostas de órteses. Principalmente em se tratando de produção pelo método de Manufatura Aditiva em máquinas de Prototipagem Rápida, que permite o emprego desses recursos com facilidade.

Com os resultados aqui alcançados, o uso desses recursos se torna mais preciso e seguro, evitando problemas com a diminuição da resistência mecânica dos aparelhos ortopédicos em questão.

\section{Orthosis and Finite Elements: a study for development of new designs through additive manufacturing}

Abstract: The gait pattern in people that present motor limitations foment the demand for auxiliary locomotion devices. These artifacts for movement assistance vary according to its shape, size and functional features, following the clinical applications desired. Among the ortheses of lower limbs, the ankle-foot orthesis aims to improve the ability to walk in people with different neuromuscular limitations, although they do not always answer patients' expectations for their aesthetic and functional characteristics. The purpose of this study is to explore the possibility of using new design in 
additive manufacturer to reproduce the shape and functional features of a ankle-foot orthesis in an efficient and modern way. Therefore, this work presents a study about the performance of the mechanical forces through the analysis of finite elements in an ankle-foot orthesis. It will be demonstrated a study of distribution of the stress on the orthopedic device in orthostatism and during the movement in the course of patient's walk.

Keywords: Additive manufacture, new designs, orthoses, finite elements.

\section{Referências bibliográficas}

B. Rogers, G. W. Bosker, M. F. Faustini, G. Walden, R. R. Neptune, R. H. Crawford. Variably Compliant Transtibial Prosthetic Socket Fabricated Using Solid Freeform 'a case study'. Journal of Prosthetics and Orthotics. V. 20. 2008.

I. Raja.; V. J. Fernandes. Reverse engineering: an industrial perspective. London: Springer Verlag, V.1 , pp. 156-179, 2008

IBGE. Censo Demográfico 2014 - Características Gerais da População. Resultados da Amostra. IBGE,2014. available inhttp://www.ibge.gov.br/ home/estatistica/populacao/cnso2014/default_populacao.shtm. Públic acess in Jul. 2017.

J. Banks. Adding value in additive manufacturing: Researchers in the United Kingdom and Europe look to 3D printing for customization. IEEE Pulse. Pages 22-26. 2013

J. H. Pallari, K. W. Dalgarno, J. Woodburn: Mass customization of foot orthoses for rheumatoid arthritis using selective laser sintering. IEEE Trans Biomed Eng. 2010

J. L. Gross. S. Y. Erkal. Lockwood. Evaluation of 3D printing and its potential impact on biotechnology and the chemical sciences. Anal Chem. Pages 3240-3253. 2014

L. Agarwal, L. Broutman, "Three-dimensional finite element analysis of spherical particlecomposites," Fibre Science and Technology, vol. 7, no. 1, pp. 63-77, 1974

L. Deberg, A. Taheri, M. Andani, M. Hosseinipour, M. Elahinia. An SMA passive ankle foot orthosis: Design, modeling, and experimental evaluation. Smart Materials Research. 2014.

L. Deberg, A. Taheri, M. Andani, M. Hosseinipour, M. Elahinia passive ankle foot orthosis: Design, modeling, and experimental evaluation. Smart Materials Research. 2014.

L. K; Gibson, S. P. Cheung. The use of rapid prototyping to assist medical applications. Rapid Prototyping Journal. Pages 53-58. 2006

L. Segerlind, Applied finite element analysis. Wiley, 1976.

M. B. Hoy. 3D printing: making things at the library. Med Ref Serv Q. Pages 94-99. 2013

M. C. Faustini, R. R. Neptune, R. H. Crawford, S. J. Stanhope. Manufacture of passive dynamic anklefoot orthoses using selective laser sintering. IEEE Trans Biomed Eng. 2008

M. Jakubinek, D. Whitman, and M. White, "Negative thermal expansion materials," Journal of Thermal Analysis and Calorimetry, vol. 99, no. 1, pp. 165-172, 2010.

M. Kelly, M. C. SpiRES, J. A. Restrepo, Orthotic and prosthetic prescriptions for today andtomorrow. Physical medicine and rehabilitation clinics of North America. V. 18. 2007

N. G. Harper, E. M. Russell, J. M. Wilken, R. R. Neptune. Selective laser sintered versus carbon fiber passive-dynamic ankle-foot orthoses: a comparison of patient walking performance. Journal of biomechanical engineering; 2014

N. Herbert, D. Simpson, W. D. Spence, W. ION. A preliminary investigation into the development of 3-D printing of prosthetic sockets. Journal of Rehabilitation Research \& Development. V.42 2005.

N. Volpato; C. H. Ahrens,; C. V. Ferreira,; G. Petrush; J. Carvalho, J. R. L. Santos, J. V. L. Silva. Prototipagem rápida: tecnologias e aplicações. São Paulo: Edgard Blucher, Pages 154-210. 2007. 
P. A. Webb: A review of rapid prototyping (RP) techniques in the medical and biomedicalsector. Journal of Medical Engineering \& Technology. V. 24. 2000.

P. F. JACOBS, Rapid prototyping \& manufacturing: fundamentals of stereolithography, Society of Manufacturing Engineers, Michigan, USA, 1992;

Pawale, V. N. Chougule, W. N. Tamboli, A. V. Mulay. Review: Analysis and Manufacturing of Ankle Foot Orthosis for Foot Drop. IOSR Journal of Mechanical and Civil Engineering (IOSR-JMCE). Pages. 12-15. 2012.

Rengier, A. Mehndiratta, L. Giesel. 3D printing based on imaging data: a review of medical applications. Int J Comput Assist Radiol Surg. 2010

S. Milusheva, E. Tosheva, D. Tochev, Y. Toshev. Personalized ankle foot orthosis with exchangeable elastic elements. Journal of Biomechanics; V.40, 2007

S. Salles, D. E. Gyi: An evaluation of personalised insoles developed using additive manufacturing. J Sports Sci. 2013

S. Salles, D. E. Gyi: The specification of personalised insoles using additive manufacturing. Work. 2012

S. Schrank, L. Hitch, K. Wallace, R. Moore, S. J. Stanhope: Assessment of a virtual functional prototyping process for the rapid manufacture of passive-dynamic ankle-foot orthoses. J Biomech Eng. 2013

S. Schrank, S. J. Stanhope. Dimensional accuracy of ankle-foot orthoses constructed by rapid customization and manufacturing framework. J. Rehabil. Res. Dev; 2011

C. Sánchez. Estudo de impacto utilizando elementos finitos e análise não linear. Masters Thesis. Universidade de São Paulo. Escola de Engenharia de São Carlos. mechanical engineering Department. 2001

T. Manee, Optimal dorsal strap placement and angulation to prevent pistoning in an ankle foot orthoses.Presented at the American Orthotic and Prosthetic Association National Assembly, Las Vegas,September 2011.

T. WHOLERS, Rapid prototyping and manufacturing: State of the industry - Annual World Wide Progress Report; 2006.

U. Gandhi, Data based models for automobile side impact analysis and design evaluation. Internationaljournal of impact engineering. V. 18, n 5, p.517 - 537 .

V. Kumar, R. Bajcsy, W. Harwin, P. Harker. Rapid design and prototyping of customizedrehabilitation aids. Communications of the ACM. V. 39, 1996.

W. Chen, F. Tang, C. Ju. Stress distribution of the foot during mid-stance to push-off in barefoot gait: a 3-D finite element analysis Clinical Biomechanics. V. 16. Pages 614-620 Aug. 2001.

X. Cui, T. Boland, D. D. D'Lima, M. K. Lotz. Thermal inkjet printing in tissue engineering and regenerative medicine. Recent Pat Drug Deliv Formul. Pages 149-155. 2012

Y. Jin, J. Plott, R. Chen, J. Wensman, A. Shih Additive Manufacturing of Custom Orthoses and Prostheses - A Review. Procedia CIRP. V. 36, Pages 199-204, 2015

3d Sytems, MATERIAL SELECTION GUIDE FOR SELECTIVE LASER SINTERING - SLS available in https://br.3dsystems.com/sites/default/files/2017-05/3D

Systems_SLS_Material\%20Selection\%20Guide_USEN_2016.12.20_WEB.pdf acess in Aug. 2017.

Healt, C. S. (2017). AFO. available in http://www.medicalexpo.es/prod/conwell medical/product68102506020.html acess in Aug. 2017.

Direct, K.-R. (2017). SmartKnit AFO Liner for Adults. available in http://www.knitritedirect.com/afosocks.html acess in Aug. 2017. 